\title{
Synthesis of control algorithms for robotic platform with electro- hydraulic drive
}

\author{
Larisa Rybak $^{1, *}$, Elena Gaponenko ${ }^{1}$, and Dmitry Malyshev ${ }^{1}$ \\ ${ }^{1}$ BSTU after V. G. Shoukhov, Dep. of machine building technology, 308012 Kostyukov str., Russia
}

\begin{abstract}
The article deals with a robotic vibroprotection platform with electro-hydraulic drive. Built mathematical model systems from the account for characteristics of the drive. Synthesized invariant controller by system state that uses reverse ties coefficients. Controller synthesized at discrete area with a restriction in the magnitude of the relative displacement of the object relative to the base. To ensure the stability of the controller are selected in regulation law amendment providing the desired value of the relative displacement. The results of mathematical modeling are presented.
\end{abstract}

\section{Introduction}

Vibration accompanies us everywhere and in most cases it is undesirable. First of all, one can call vibration and fluctuations in road and rail transport, in the mining industry, vibration of equipment, motors and machines, oil and gas platforms, buildings and structures in the zone of high seismic hazard, undesirable vibrations of laboratory tables (especially optical ones) etc. In all these cases, the task is to isolate the person or the payload from the source of vibration or, conversely, isolate the source of vibration. Particularly important today is the issue of human vibroprotection (including operator-driver of mobile machines and equipment).

General vibration, as a result of the mechanical interaction of a person with the external environment, is an important ecological factor throughout biological evolution and has great biological activity. The formation of physiological and pathological shifts on the part of various systems of the body, the frequency and severity of these disorders depends on the ergonomic data of the workplace and human characteristics.

The nature and depth of vibration disorders are caused by changes in the vestibular analyzer, musculoskeletal system, cardiovascular system, hemodynamics of blood circulation, neurohumoral sphere. Sensitive to vibration is the opto-vestibulospinal system with the development of the main clinical manifestations, such as a headache, non-systemic dizziness, impaired coordination, lability of arterial pressure, changes in the vessels of the fundus, signs of the neurosis-like syndrome and autonomic dysfunction, pain in the back muscles, lumbar spine. Vibration causes a decrease in visual acuity, a violation of color perception, a narrowing of the field of view, a decrease in the stability of clear vision, a decrease in functional mobility, a disorder in fixing objects with the eye, a violation of the sharpness of the perception of objects, a difficulty in reading instrumental information.
The level and frequency spectrum of vibration in the driver's workplace depends on the road profile, driving speed, engine vibration level, chassis and cabin suspension and, finally, the driver's seat. For example, the vibration levels of the driver's seats of excavators ECG-8I, ECG-4,6B in the quarry of the Lebedinsky ore mining and processing combine exceed permissible by 2 $\ldots 10 \mathrm{~dB}$ in the frequency range $2 \ldots 16 \mathrm{~Hz}$. Spectral characteristics of input excitations of operators' workplaces show that the vibrational energy of quarry machines is concentrated at the lower end of the frequency range and has characteristic peaks.

In many respects, the effect of vibration on a person depends on its spectral composition. Areas of frequencies are known in which vibration levels can affect the performance of production operations. So the frequency of $4 \ldots 5 \mathrm{~Hz}$ is a resonant area for a person working standing, $11 \mathrm{~Hz}$ - sitting.

Reducing the level of vibration of workplaces and improving their ergonomic characteristics will solve one of the most important problems of improving working conditions and preserving the health of workers.

Methods of protection from vibration are divided into two groups: methods that reduce the parameters of vibration by influencing the source of excitation, and methods that reduce the parameters of vibration along the path of its propagation from the source of excitation.

Vibration isolation system is divided into active and passive. Despite all the structural differences, the essence of the vibration damping system is the same. The passive system consists of a spring and a damper. The spring is designed to soften vibrations and shocks, and the damper - to quench the fluctuations that have arisen in the system. The active system also uses an additional pair consisting of an accelerometer and an electromagnetic drive, which makes it possible to achieve an exceptionally high degree of vibration isolation.

\footnotetext{
* Corresponding author: rl_bgtu@intbel.ru
} 
It is proposed to develop an active system that provides effective vibration isolation from $0.1 \mathrm{~Hz}$, the absence of resonances and the necessary rigidity under the action of constant accelerations.

\section{Description of schemes and statement of the problem}

Consider a system with an electrohydraulic actuator (Figure 1), which includes a hydraulic cylinder located between the object and the base, an electrohydraulic transducer, as well as the actual vibration protection object, accelerometers on the object and the base, a relative displacement sensor and a controller.

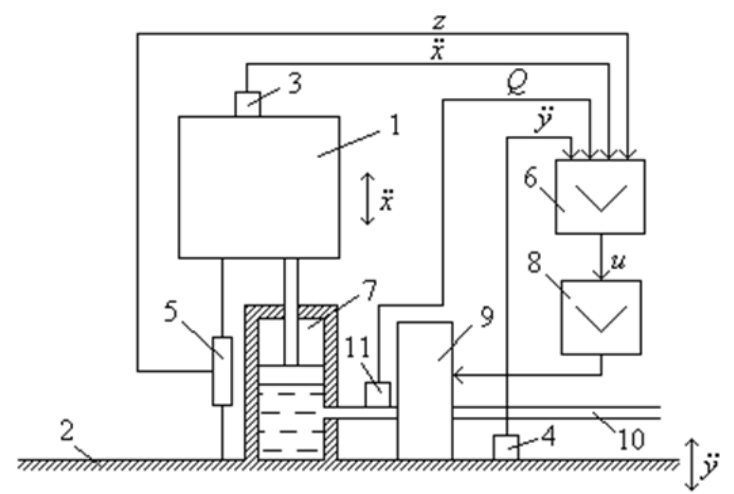

Fig. 1. System with an electrohydraulic actuator 1 vibroprotection object; 2 - base; 3, 4 - accelerometers on the object and the base; 5 - a sensor of the relative displacement; 6 - a controller; 7 - hydraulic cylinder; 8 - power amplifier; 9 electro-hydraulic converter; 10 - hydraulic line; 11 - working fluid flow sensor

The electrohydraulic conversion unit consists of a throttling distributor with a stepping motor which, through a screw pair, moves the four-spool valve in the axial direction. The stepper motor supplied voltage pulses, and each pulse corresponds to the rotation of its shaft at a certain angle-pitch (angular increments), which often is $1,5^{\circ}$. Thus, the angle of rotation is determined by the number of pulses fed, and the rotational frequency by the frequency of their following. The throttle valve is essentially a hydraulic power amplifier that converts the input mechanical action into a corresponding displacement of the output link, which allows the flow and direction of the oil flow to be varied in several lines simultaneously, depending on the external control action.

Consider the synthesis of invariant control $[1,2]$, which will create such a system operating modes which provide full stabilization vibration isolation object [3].

The problem of the synthesis of invariant control is essentially a task of synthesizing an algorithm for controlling the inverse dynamic problem. The basis of the method of inverse problems of dynamics is the following: the algorithm for the normalization of the control function is constructed by the principle of symmetry of the structure and inversion of operations with respect to the structure and group of operations corresponding to the mathematical model of the controlled process. Consequently, the symmetry properties inherent to the object and a control system that uniquely define the structure and parameters of the algorithm of forming control functions assigned to the path of movement $\mathbf{X}^{*}(t)$ the structure and parameters of the control algorithm be determined by the structure and parameters of a mathematical model of the object.

This provision is the methodological basis for constructing motion control algorithms for dynamic systems. In this case, in fact, the task of designing the control algorithm is presented as the inverse problem of dynamics: it is required to find a control function (power) $u^{*}$, which implements the prescribed trajectory of motion $\mathbf{X}^{*}$ [4].

Let us consider the procedure for the synthesis of an invariant control perturbation applied to the system under consideration with an electrohydraulic actuator. We describe the entire system, including the actuator, in the state space. In developing the mathematical model of this executive mechanism, a system of equations

$$
\left\{\begin{array}{l}
\ddot{z}=\frac{A k_{\text {тр }}}{m} p, \\
\dot{p}=-\frac{4 A E}{V} \dot{z}-\frac{4 E k_{\mathrm{yT}}}{V} p+\frac{4 E}{V} Q
\end{array}\right.
$$

We introduce 2 state variables: $x_{1}=\dot{z}, x_{2}=p$ The system of equations (1) becomes

$$
\left\{\begin{array}{l}
\dot{x}_{1}=\frac{A k_{\text {тр }}}{m} x_{2} \\
\dot{x}_{2}=-\frac{4 A E}{V} x_{1}-\frac{4 E k_{\text {ут }}}{V} x_{2}+\frac{4 E}{V} Q
\end{array}\right.
$$

or in vector-matrix form

$$
\dot{\mathbf{X}}=\mathbf{A X}+\mathbf{B} Q
$$

Synthesis optimal controller problem will be solved in the discrete area, to which the system of equations (3) translate into discrete form

$$
\mathbf{X}[i+1]=\mathbf{A}_{\Delta} \mathbf{X}[i]+\mathbf{B}_{\Delta} Q[i]
$$

$$
\begin{aligned}
& \text { where } \mathbf{A}_{\boldsymbol{\Delta}}=\exp (\mathbf{A} T)=\mathbf{I}+\sum_{i=1}^{\infty} \frac{\mathbf{A}^{i} T^{i}}{i !}, \\
& \mathbf{B}_{\boldsymbol{\Delta}}=\left(\int_{0}^{T} \exp (\mathbf{A} T) d t\right) \mathbf{B}=\left(\mathbf{I} T+\sum_{i=1}^{\infty} \frac{\mathbf{A}^{i} T^{i+1}}{(i+1) !}\right) \mathbf{B} .
\end{aligned}
$$

In the equations (4), the symbols $\mathbf{A}_{\Delta}$ and $\mathbf{B}_{\Delta}$ designate analogs of matrices $\mathbf{A}$ and $\mathbf{B}$ for a discrete system, and the symbol $T$ is entered to designate the sampling period. Because of the high complexity of mathematical calculations is not appropriate to display the general form and the matrices $\mathbf{A}_{\Delta}$ and $\mathbf{B}_{\Delta}$. Preferred to calculate the matrix $\mathbf{A}_{\Delta}$ and $\mathbf{B}_{\Delta}$ in numerical form for each specific pair of matrices $\mathbf{A}$ and $\mathbf{B}$. Next, to simplify the entries, we return to the notation without indices: $\mathbf{A}=\mathbf{A}_{\Delta}$; $\mathbf{B}=\mathbf{B}_{\Delta}$; equation

From the system equation I (1) can obtain the

$$
\ddot{x}[i]-\ddot{y}[i]=\frac{A k_{\text {тр }}}{m} p[i]
$$


Having put $\ddot{x}[i]=0$, You can obtain an expression for the desired differential etc. The pressure in the hydraulic cylinder::

$$
p[i]=-\frac{m}{A k_{\text {тр }}} \ddot{y}[i]
$$

Let $\mathbf{A}=\left[\begin{array}{ll}a_{11} & a_{12} \\ a_{21} & a_{22}\end{array}\right], \quad \mathbf{B}=\left[\begin{array}{l}b_{1} \\ b_{2}\end{array}\right]$, where $a_{i j}, \quad b_{k}-$ specific numerical values obtained for a particular system after its reduction to form (4). Then from II system equation (4) we obtain

$$
Q[i]=\frac{1}{b_{2}} p[i+1]-\frac{a_{22}}{b_{2}} p[i]-\frac{a_{21}}{b_{2}} \dot{z}[i]
$$

In view of (6), (7) we obtain the value of the manipulated variable flow

$$
u_{Q}[i]=-\frac{m}{A k_{\mathrm{rp}} b_{2}} \ddot{y}[i+1]+\frac{m a_{22}}{A k_{\mathrm{rp}} b_{2}} \ddot{y}[i]-\frac{a_{21}}{b_{2}} \dot{z}[i]
$$

For formation of the algorithm of the control law (8) must be the acceleration value based on the following clock cycle $\ddot{y}[i+1]$. It is possible to use a method of predicting a given value, as proposed in $[3,6]$, which is to use extrapolation Lagrange formula

$$
\hat{y}[i+1]=3 \ddot{y}[i]-3 \ddot{y}[i-1]+\ddot{y}[i-2]
$$

Using Lagrange estimation, considering the formulas (8) and (9), to form the expression control law

$$
\begin{aligned}
u_{Q}[i]= & \frac{m\left(a_{22}-3\right)}{A k_{\mathrm{Tp}} b_{2}} \ddot{y}[i]+\frac{3 m}{A k_{\mathrm{Tp}} b_{2}} \ddot{y}[i-1]- \\
& -\frac{m}{A k_{\mathrm{Tp}} b_{2}} \ddot{y}[i-2]-\frac{a_{21}}{b_{2}} \dot{z}[i]
\end{aligned}
$$

We synthesize the control system for the electrohydraulic system.

The matrices $\mathbf{A}$ and $\mathbf{B}$ of the equation (3) of equation (3) for such a following system:

$$
\begin{gathered}
\mathbf{A}=\left[\begin{array}{cc}
0 & 3,656 \times 10^{-5} \\
-2,014 \times 10^{10} & -9,680 \times 10^{3}
\end{array}\right] \\
\mathbf{B}=\left[\begin{array}{c}
0 \\
5,232 \times 10^{12}
\end{array}\right] .
\end{gathered}
$$

Going to a discrete task, taking a sampling period $T=200 \mathrm{~ms}$, we get the following matrix $\mathbf{A}_{\Delta}$ and $\mathbf{B}_{\Delta}$ equation (4):

$$
\begin{gathered}
\mathbf{A}_{\boldsymbol{\Delta}}=\left[\begin{array}{cc}
0,992 & 3,217 \times 10^{-9} \\
-1,772 \times 10^{6} & 0,140
\end{array}\right], \\
\mathbf{B}_{\Delta}=\left[\begin{array}{c}
2,201 \\
4,604 \times 10^{8}
\end{array}\right] .
\end{gathered}
$$

After performing the appropriate calculations, we define the control law for the invariant controller

$$
\begin{gathered}
u_{Q}[i]=\left(-1,699 \times 10^{-4}\right) \ddot{y}[i]+\left(1,782 \times 10^{-4}\right) \ddot{y}[i-1]+ \\
\left(-5,941 \times 10^{-5}\right) \ddot{y}[i-2]+\left(3,848 \times 10^{-3}\right) \dot{z}[i]
\end{gathered}
$$

To control algorithm (11), the mathematical modeling of the system when harmonic distortion. Graphs of acceleration at the object and displacement of the object relative to the base for the frequency of the disturbing effect $f=2 \mathrm{~Hz}$ are shown in Fig. 2. The amplitude of the disturbance is assumed equal to $A_{\ddot{y}}=g$. In this case, the disturbance is almost completely suppressed, if we do not take into account the noise caused by the stepping nature of the electric motor.

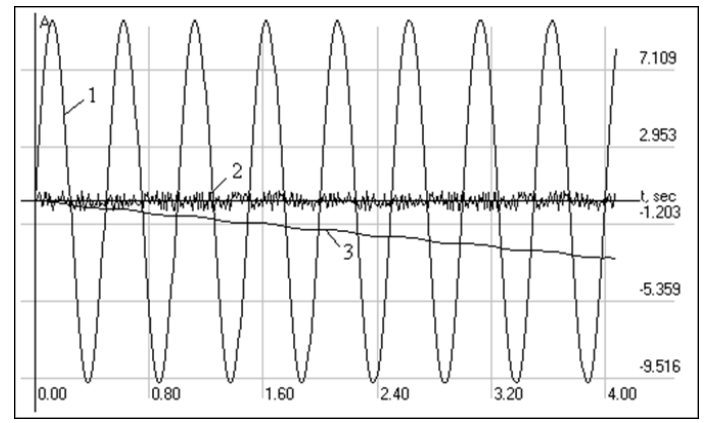

Fig. 2. Modeling the system operation at the disturbance frequency $f=2 \mathrm{~Hz}: 1$ - basis acceleration; 2 - object acceleration; 3 - moving the object relative to the base.

According to the expression (10), a control law there is only one value being a coordinate system state - is the relative velocity of the object $\dot{z}$. Can be introduced into the control channel of the relative speed of the object defining a small correction $\Delta[5]$, whereas the control law takes the form

$$
\begin{gathered}
u_{Q}[i]=\frac{m\left(a_{22}-3\right)}{A k_{\mathrm{tp}} b_{2}} \ddot{y}[i]+\frac{3 m}{A k_{\mathrm{tp}} b_{2}} \ddot{y}[i-1]- \\
-\frac{m}{A k_{\mathrm{Tp}} b_{2}} \ddot{y}[i-2]-(1-\Delta) \frac{a_{21}}{b_{2}} \dot{z}[i]
\end{gathered}
$$

The introduction of an amendment to the nature of the impact on the system is equivalent to introducing restrictive feedback into the control channel at the relative velocity of the object. When $\Delta=0$ expression (12) gives a control law for a perfect invariant controller.

In addition, the control signal must be limited from above and from below in accordance with expression

$u_{Q}^{\prime}[i]= \begin{cases}u_{Q}[i], & \left|u_{Q}[i]\right| \leq Q_{\max }, \\ \operatorname{sgn}\left(u_{Q}[i]\right) Q_{\max }, & \left|u_{Q}[i]\right|>Q_{\max },\end{cases}$

where $Q_{\max }$ is maximum allowable working fluid flow, defined by parameters of the throttle control valve and hydraulic lines. As before, in the control loop for the flow of working fluid as a controller, we select a threeposition relay controller.

In Fig. 3 is a structural diagram of a vibroprotection system invariant controller taking into account the control law (12), and the constraints on the manipulated variable. 


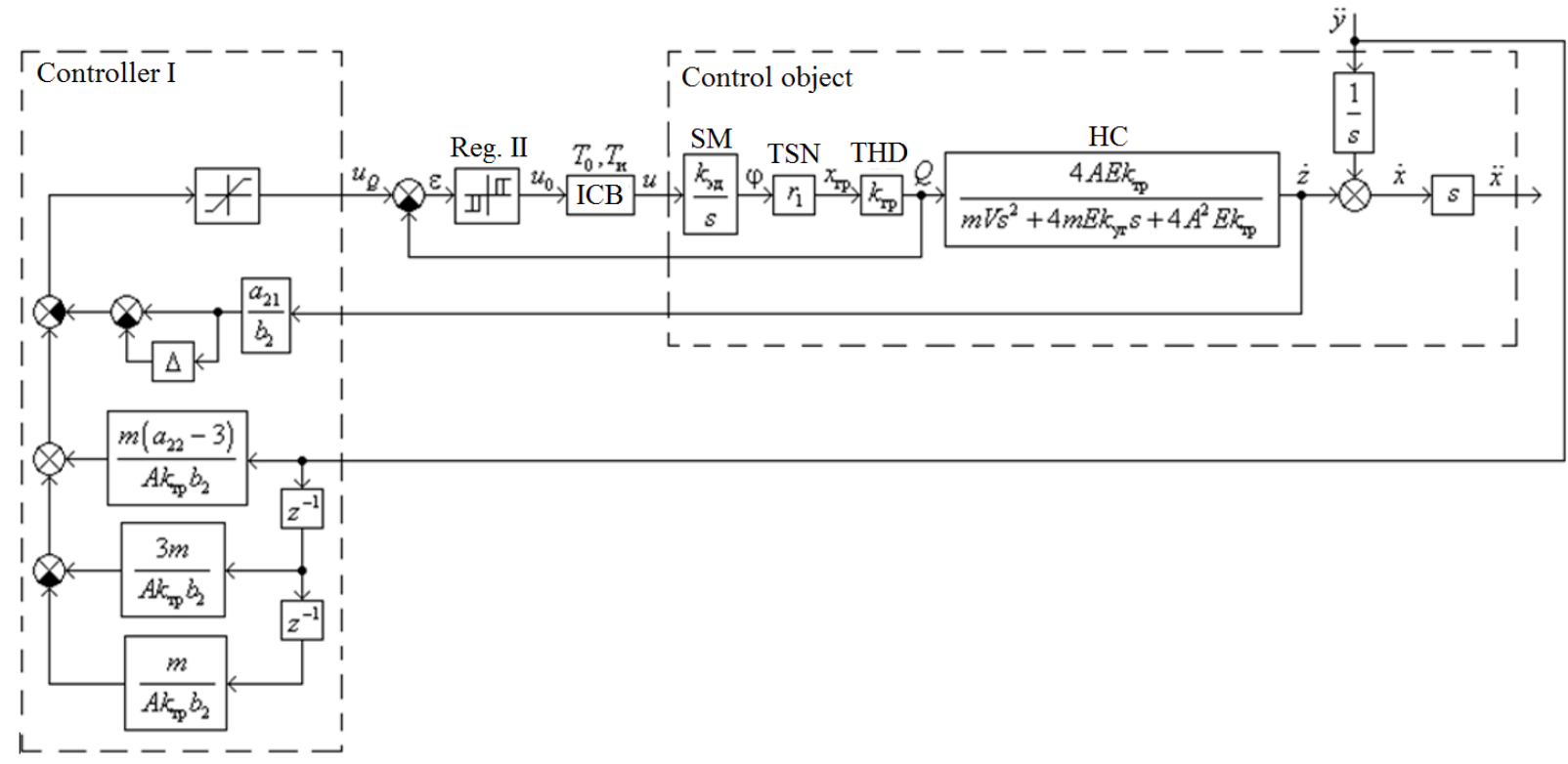

Fig. 3. Active vibroprotection system structural diagram with the electro-hydraulic actuating mechanism and invariant controller: SM - stepper motor, TSN - transmission "screw-nut", THD - throttling hydraulic distributor, HC - hydraulic cylinder.

For the control algorithm (1.131) (the magnitude of the correction $\Delta=0,01)$ a simulation of the operation of the system was carried out under perturbations of various types. Graphs of acceleration at the object and displacement of the object relative to the base for the frequency of the disturbing effect $f=1 \mathrm{~Hz}$ are shown in Fig. 4.

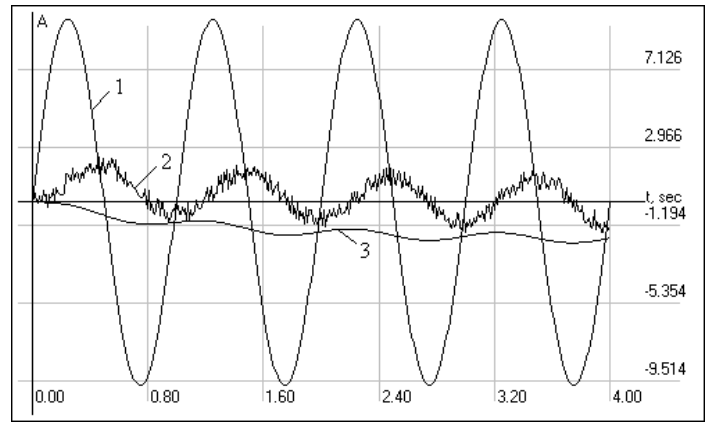

Fig. 4. Simulation of the system at a frequency of $1 \mathrm{~Hz}$ disturbance.

The amplitude of the disturbing effect was assumed equal to $A_{\ddot{y}}=g$. In this case, the coefficient of suppression of the disturbing vibrational action in amplitude is $\frac{A_{\ddot{x}}}{A_{\ddot{y}}}=0,2$. It should also be noted that the relative movement of the object is limited and can be kept at an acceptable level. Level steady relative movement can be reduced by increasing the correction $\Delta$ a control law (12). However, it should be borne in mind that in this case, the acceleration amplitude on the object will increase. In any case, the decision will have a compromised character.

In the course of mathematical modeling, it was found that disturbances in the range $1 \ldots 20 \mathrm{~Hz}$ are suppressed by a system with an invariant controller better than a system with an optimal controller. This means that with the same level of relative movement of the object and comparable control costs, it is possible to achieve a somewhat lower level of acceleration at the site. However, in general, the frequency range of operation of the system in the case of using the invariant controller narrows.

This work was supported by the Russian Science Foundation, the agreement number 16-19-00148.

\section{References}

1. F.A. Aliyev. V.B. Larin, K.I. Naumenko, V.N.Suntsev, Optimization of time-invariant control systems, 327 (1978)

2. A.V. Gradetsky, A.I. Pashkov, L.A. Rybak, A.V. Sinev, V.S. Solovyov, Probl. of mach. build. and mach. reliab., 4, 78-86 (1995)

3. L.A. Rybak, A.V. Sinev, A.I. Pashkov, The synthesis of the active vibration isolation systems in space objects, 160 (1997)

4. E.A. Fedosov, A.A. Krasovsky, E.P. Popov et al., Machine building, 1-4, 688 (2000)

5. L.A. Rybak, V.K. Arshinov, R.V. Erzhukov, XIII Int. Symp. "Dyn. of vib-imp. (hi. nonl.) sys.", 82 (2001)

\footnotetext{
Corresponding author: rl_bgtu@intbel.ru
} 